\title{
Quantum Engineering of InAs/GaAs Quantum Dot Based Intermediate Band Solar Cells
}

\author{
Neil S. Beattie, ${ }^{* \dagger}{ }^{\dagger}$ Patrick See, ${ }^{\ddagger}$ Guillaume Zoppi, ${ }^{\dagger}$ Palat M. Ushasree, ${ }^{\dagger}$ Martial Duchamp, ${ }^{\S}$ Ian Farrer,
} David A. Ritchie, ${ }^{\perp}$ and Stanko Tomić ${ }^{*}, \# \mathbb{C}$

\author{
${ }^{\dagger}$ Department of Mathematics, Physics and Electrical Engineering, Northumbria University, Newcastle upon Tyne, NE1 8ST, United \\ Kingdom \\ ${ }^{\ddagger}$ National Physical Laboratory, Teddington, TW11 0LW, United Kingdom \\ ${ }^{\S}$ School of Materials Science and Engineering, Nanyang Technological University, Singapore 639798, Singapore \\ "Department of Electrical and Electronic Engineering, University of Sheffield, Sheffield, S1 3JD, United Kingdom \\ ${ }^{\perp}$ Cavendish Laboratory, University of Cambridge, Cambridge, CB3 0HE, United Kingdom \\ \#Joule Physics Laboratory, School of Computing Science and Engineering, University of Salford, Manchester M5 4WT, United \\ Kingdom
}

\section{Supporting Information}

\begin{abstract}
The efficiency of a solar cell can be substantially increased by opening new energy gaps within the semiconductor band gap. This creates additional optical absorption pathways which can be fully exploited under concentrated sunlight. Here we report a new approach to opening a sizable energy gap in a single junction GaAs solar cell using an array of InAs quantum dots that leads directly to high device open circuit voltage. High resolution imaging of individual quantum dots provides experimentally obtained dimensions to a quantum mechanical model which can be used to design an optimized quantum dot array. This is then implemented by precisely engineering the shape and size of the quantum dots resulting in a total area (active area) efficiency of $18.3 \%$ $(19.7 \%)$ at 5 suns concentration. The work demonstrates that

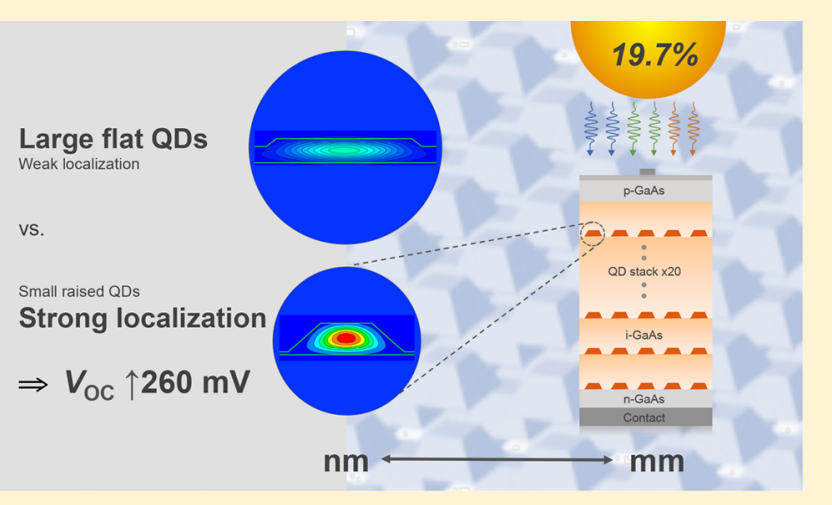
only the inclusion of an appropriately designed quantum dot array in a solar cell has the potential to result in ultrahigh efficiency under concentration.
\end{abstract}

KEYWORDS: intermediate band solar cell, quantum dots, photovoltaics, concentrated sunlight

$\mathrm{T}$ he cost of electricity produced from a photovoltaic system depends directly on the efficiency of the solar cells. Therefore, increasing solar cell efficiency is a key driver of innovation which gives rise to the category of ultrahigh efficiency solar cells. ${ }^{1}$ This category includes performance that exceeds the equivalent single $p n$-junction solar cell, governed by the fundamental Shockley-Queisser limit. ${ }^{2}$ A particular technology which offers this potential is the intermediate band solar cell $(\text { IBSC) })^{3,4}$ which captures a greater fraction of the incident solar spectrum via a narrow band of electronic states within the semiconductor band gap. Moreover, this benefit can be fully exploited under concentrated sunlight where, in contrast to a single energy gap solar cell, the device short circuit current $J_{\mathrm{sc}}$ increases superlinearly.

For the IBSC to function correctly, the semiconductor valence band (VB), intermediate band (IB) and conduction band (CB) must all be electronically isolated. This requires well-separated quasi-Fermi levels under external illumination and pure zero density of states (DOS) between the IB and CB. ${ }^{5}$
Under ideal conditions, IBSC theory predicts a maximum efficiency of $63 \%$ for an energy separation $E_{\mathrm{VB}, \mathrm{IB}}=1.2 \mathrm{eV}$ between the $\mathrm{VB}$ and the $\mathrm{IB}$, and $E_{\mathrm{IB}, \mathrm{CB}}=0.7 \mathrm{eV}$ between the IB and the $\mathrm{CB} .{ }^{3}$ The most studied experimental IBSC prototype is based on incorporating an array of self-assembled InAs semiconductor quantum dots within the intrinsic region of a GaAs pin diode. ${ }^{6-12}$ This gives rise to the quantum dot intermediate band solar cell (QD-IBSC). Although $E_{\mathrm{IB}, \mathrm{CB}}$ is less than ideal $(\sim 5-120 \mathrm{meV})$ for this material system, a realistically achievable solar energy conversion of $34 \%$ under concentration is nevertheless predicted ${ }^{13}$ and it is notable that the first demonstration of a photovoltaic concentrator module using QD-IBSCs was recently reported. ${ }^{14}$

In this work we present a new experimental approach to opening a clear energy gap between an IB of quantum states

Received: June 26, 2017

Published: October 13, 2017 
and the CB in a QD-IBSC. By precisely controlling the shape and size of the quantum dots in the quantum dot array used to create the IB we can, for the first time in an IBSC implement a quantum mechanical design which simultaneously fulfils the objectives of IB regime operation and high device open circuit voltage $\left(V_{\text {oc }}\right)$ without the need for additional wider band gap barrier layers. The findings provide a pathway to high efficiency InAs QD-IBSCs and an approach to creating ultrahigh efficiency III-V multijunction solar cells by virtue of the compatibility of the technologies. ${ }^{15}$

Solar cell device wafers were grown via MBE and included 20 stacked layers of InAs self-assembled quantum dots in the intrinsic region of a GaAs pin diode. Here, we capitalize on the quality and precision of $\mathrm{MBE}$ growth to create quantum dot arrays that do not require strain compensation. ${ }^{16,17}$ Furthermore, transferring a technique from light emitting applications ${ }^{18}$ affords control over the shape and size of the quantum dots at the nanometre scale. ${ }^{19}$ It therefore offers a way to engineer the discrete energy levels within the quantum dots and ultimately the separation between the IB and the CB. We refer to this growth technique as quantum engineering. More specifically, quantum dot growth proceeds initially by the well-established Stranski-Krastanov growth with the formation of a $2 \mathrm{D}$ InAs wetting layer before it becomes energetically more favorable to form InAs quantum dots. Typically these dots are lens-shaped and remain so for a relatively cold growth temperature used to create $35 \mathrm{~nm} \mathrm{GaAs} \mathrm{cap} \mathrm{(spacer)} \mathrm{layer.} \mathrm{If}$ instead, only the first few monolayers of GaAs are grown cold before the temperature is elevated, the exposed In desorbs from the upper layers of a given quantum dot. The net result is a smaller quantum dot with a truncated pyramid structure. Controlling the temperature profile and the spacer layer depth (near atomic precision) at which such quantum engineering is applied, allows the fabrication of highly uniform arrays containing different sizes of quantum dots.

Consider, for example, the high resolution high-angle annular-dark-field (HAADF) scanning transmission electron microscopy (STEM) images in Figure 1 of individual quantum dots from a reference QD-IBSC (Figure 1a-c) and a quantum

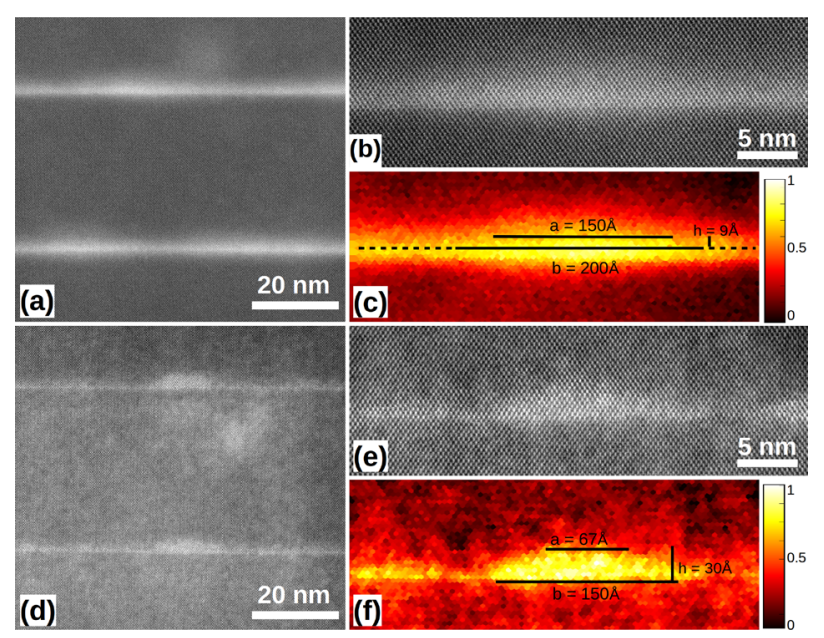

Figure 1. (a, b, d, e) HAADF-STEM images and (c, f) local mean intensity maps of individual quantum dots $b$ and e, respectively. $(a-c)$ QD-IBSCs without and $(\mathrm{d}-\mathrm{f})$ with quantum engineering. The drawn truncated pyramids in (c) and (f) are the estimated $2 \mathrm{D}$ projection shape of the quantum dots and used to estimate the size of the quantum dots. engineered QD-IBSC (Figure 1d-f). The full device structure for each of these wafers may be found in the Supporting Information (Figures $\mathrm{S} 1-\mathrm{Sn}$ and $\mathrm{S} 2-\mathrm{Sn}$ ). The imaging conditions were chosen to acquire HAADF-STEM images which are approximately proportional to the square of the atomic number. Further, the white contrast indicates the presence of a large indium concentration compared to the surrounding GaAs matrix. In order to better visualize the variation of indium concentration, the local mean intensity of the atomic dumbbells were computed using a Voronoi polygon centered on the local intensity maxima (Figure $1 \mathrm{c}, \mathrm{f}$ ). ${ }^{20}$ The projected dimensions of the quantum dots are extracted from the local mean intensity maps which are bounded by a truncated pyramid shape with quantum dot base length $b$, length at the site of truncation $a$, and height $h$. Note that the height of the QDs, $h$, has been estimated from the top of the wetting layer and the top of the QD in Figure 1c and between the bottom and the top of the QD in Figure if due to almost negligible WL. Such estimated QDs dimensions have been passed to the simulations. From these data the extracted dimensions are, $a=150 \AA, b=200 \AA$, and $h=9 \AA$, and $a=67$ $\AA, b=150 \AA$, and $h=30 \AA$, for the reference and quantum engineered QD-IBSC, respectively.

Using the quantum dot shape and size dimensions obtained from the STEM data, we modeled the quantum dot arrays in the reference QD-IBSC and the quantum engineered QD-IBSC using a combination of a $\mathbf{k} \cdot \mathbf{p}$ method combined with periodic boundary conditions in the vertical direction and described in greater detail elsewhere. ${ }^{5,13,21}$ In Figure 2a,b we plot the contours of the charge densities of the electron ground state $\left(e_{0}\right)$, first excited state $\left(e_{1}\right)$, and the hole ground state $\left(h_{0}\right)$ for the two quantum dot arrays. Here $e_{0}, e_{1}$, and $h_{0}$ correspond to the $\mathrm{IB}, \mathrm{CB}$, and $\mathrm{VB}$, respectively. It is evident that the quantum engineered QD-IBSC (Figure 2a) results in significant localization of $e_{0}, e_{1}$, and $h_{0}$ in the quantum dot, while for the reference QD-IBSC (Figure 2b), a significant part of the charge density is actually localized in the wetting layer. In this case, the whole layer behaves more like a quantum well with modulated $z$ dimension.

Figure $2 \mathrm{c}, \mathrm{d}$ shows the single particle energy spectra for the two structures. The quantum engineered QD-IBSC exhibits a clear energy separation between $e_{0}$ and $e_{1}$ states of $\Delta E_{\mathrm{e} 0 \mathrm{e} 1}=83$ $\mathrm{meV}$ which is more than twice that of the reference QD-IBSC, where $\Delta E_{\mathrm{e} 0, \mathrm{e} 1}=30 \mathrm{meV}$ and also more than three times the thermal energy $\left(k_{\mathrm{B}} T=26 \mathrm{meV}\right)$ at room temperature. This is clear indication that the quantum engineered QD-IBSC will provide much better conditions for the formation of the quasiFermi level separation between the IB and CB under external illumination.

In order to clearly identify the contribution of the IB to the optical properties of the two quantum dot arrays in the quantum engineered QD-IBSC and the reference QD-IBSC, we calculate their absorption spectra within the dipole approximation as $5,13,21$

$$
\begin{aligned}
\alpha(\hbar \omega)= & \frac{\pi e^{2}}{c \epsilon_{0} m_{0}^{2} \bar{n} \omega \Omega} \\
& \times \sum_{\mathbf{K}} \sum_{\mathrm{i}, \mathrm{f}}|\langle i|\hat{e} \cdot \mathbf{p}(\mathbf{K})| f\rangle|^{2} \delta\left[E_{\mathrm{f}}(\mathbf{K})-E_{\mathrm{i}}(\mathbf{K})-\hbar \omega\right]\left(f_{\mathrm{i}}-f_{\mathrm{f}}\right)
\end{aligned}
$$

where $e$ is the electron charge, $\bar{n}$ is the refractive index, $\epsilon_{0}$ is the dielectric permittivity of vacuum, $c$ is the speed of light, $\Omega$ is the volume of the structure, $\hat{\mathbf{e}}$ is the unit vector of light polarization, 

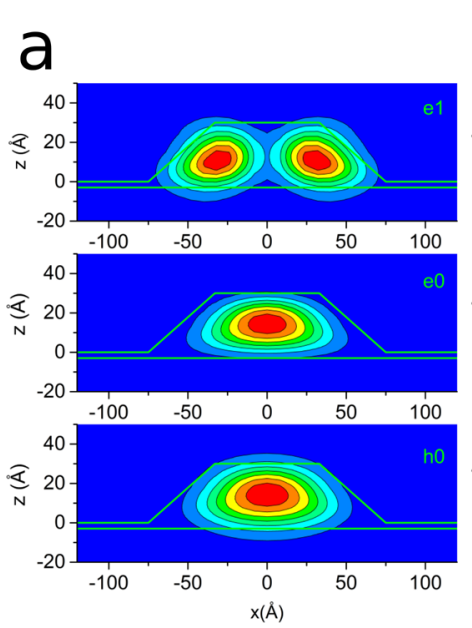
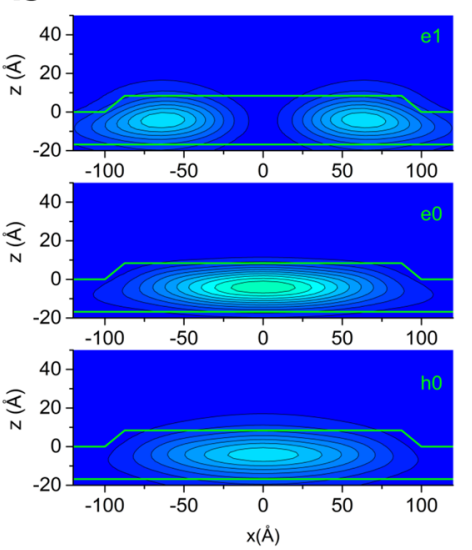

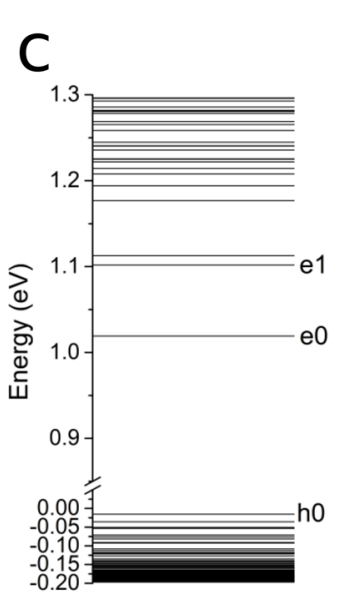

Figure 2. (a, b) Charge densities and (c, d) electronic structure for individual quantum dots calculated using dimensions obtained from STEM measurements. $(\mathrm{a}, \mathrm{c})$ correspond to a quantum dot from a quantum engineered array while $(\mathrm{b}, \mathrm{d})$ correspond to a reference array. $e_{0}$ and $e_{1}$ denote ground and first excited states in the $\mathrm{CB}$ and $h_{0}$ is the ground state in the VB.

and $\mathbf{p}(\mathbf{K})$ is the momentum operator. The optical dipole matrix element is given by $\langle i|\hat{\mathbf{e}} \cdot \mathbf{p}(\mathbf{K})| f\rangle$, and varies inside the $1 \mathrm{D}$ Brillouin zone (BZ) of an QD array. The initial and final state energies are $E_{\mathrm{i}}(\mathbf{K})$ and $E_{\mathrm{f}}(\mathbf{K})$, which also varies throughout the QD array $\mathrm{BZ}, \hbar \omega$ is the photon energy, and $f_{\mathrm{i}(\mathrm{f})}$ is the initial (final) state Fermi-Dirac distribution function.

Figure 3 shows two absorption spectra for each array: one between all VB and CB states (solid line) and one between all

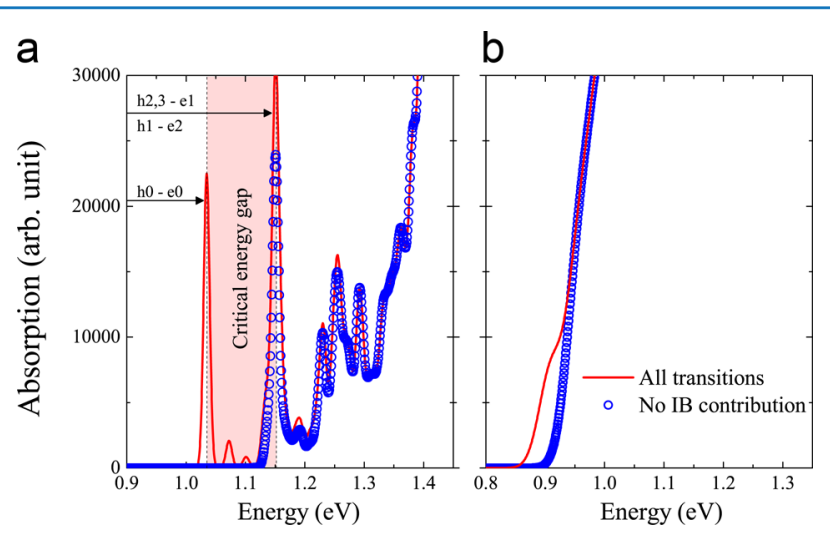

Figure 3. Absorption spectra of quantum dot arrays corresponding to (a) a quantum engineered QD-IBSC and (b) a reference QD-IBSC. The solid line accounts for optical transitions between all states in the $\mathrm{CB}$ and $\mathrm{VB}$ while the open circles indicate the case for which transitions originating from $\mathrm{e} 0$ have been subtracted. Labels and horizontal arrows mark the dominate transitions that contribute to particular absorption peaks.

$\mathrm{VB}$ and $\mathrm{CB}$ states except those originating from $\mathrm{e}_{0}$ (open circles). It is evident that for the array corresponding to the quantum engineered QD-IBSC (Figure 3(a)) clear regions of zero DOS exists between $\mathrm{e} 1$ and $\mathrm{e} 0$. This is the signature of an energetically isolated IB.

In contrast, however, for the array corresponding to the reference QD-IBSC (Figure 3b) strong hybridization exists between all states in the $\mathrm{CB}$ and there is no clear opening of a second energy gap associated with an IB. Due to such strong hybridization of the states, the effect of the quantum dot array appears only as a slight shoulder in the data which pushes the absorption edge to lower energy. Note that this shoulder would be even less pronounced had $h$ been measured from the bottom of the InAs wetting layer thus the value of $\Delta E_{\mathrm{e} 0, \mathrm{e} 1}$ may be taken as conservative. This behavior has been been experimentally observed by several groups, (see Review ${ }^{22}$ ), but unfortunately the resulting increase in $J_{\mathrm{sc}}$ comes at the expense of significant reduction in $V_{\text {oc }}$ due to the loss of IB features, that is, loss of the quasi-Fermi levels separation between IB and $\mathrm{CB}$ under illumination. This has been a key bottleneck in the development of the QD-IBSC technology and our work demonstrates that the advantages of the IBSC are indeed accessible in this system if the quantum dot array is appropriately designed.

Figure $4 \mathrm{a}$ shows the external quantum efficiency (EQE) spectra for a GaAs control solar cell, a reference QD-IBSC and a quantum engineered QD-IBSC. Note here that the reference QD-IBSC and the quantum engineered QD-IBSC are constructed from arrays of the quantum dots shown in Figure $1 \mathrm{c}$ and $\mathrm{f}$, respectively. A larger range of EQE for these solar cells is presented elsewhere ${ }^{23}$ and here we focus on the region beyond the GaAs band edge. As expected, the EQE signal for the GaAs control solar cell falls sharply at the GaAs band edge, while the EQE for the QD-IBSCs extends to longer wavelengths. This behavior is attributed to VB to IB optical transitions. ${ }^{21}$ The important point to note here, however, is the difference between the EQE data for the quantum engineered QD-IBSC compared with the reference QD-IBSC. While both solar cells exhibit a InAs wetting layer characteristic around 1.35 $\mathrm{eV}$, only the quantum engineered QD-IBSC presents a shoulder at approximately $1.19 \mathrm{eV}$. From room temperature electroluminescence (EL) measurements (also shown in Figure $4 a)$, we determined this to be the first excited state el. The contribution of the ground states in the EQE is beyond the range of the detector, however, the difference between e 1 and the ground state transition $\mathrm{e} 0-\mathrm{h} 0$ determined from $\mathrm{EL}$ is 70 $\mathrm{meV}$ which is in good agreement with our theory.

In parallel with our quantum engineering technique, we optimized the device structures to further increase performance. This included the addition of an AlGaAs window layer (see Figure $\mathrm{S} 2-\mathrm{Sn}$ ), which reduces surface recombination. Figure $4 \mathrm{~b}$ shows the 1 sun $\left(100 \mathrm{~mW} / \mathrm{cm}^{2}\right) J V$ characteristics for the three devices. Both the GaAs control and the quantum engineered QD-IBSC included a window layer and this is clearly evident as a significant increase in $J_{\mathrm{sc}}$ which is $18.3,18.9$, 
a

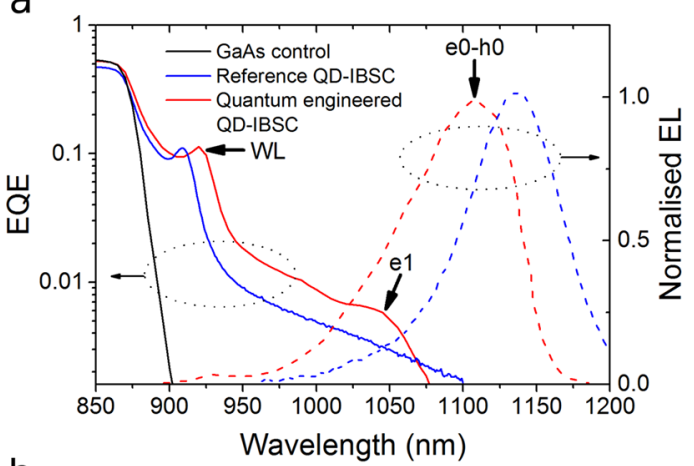

b

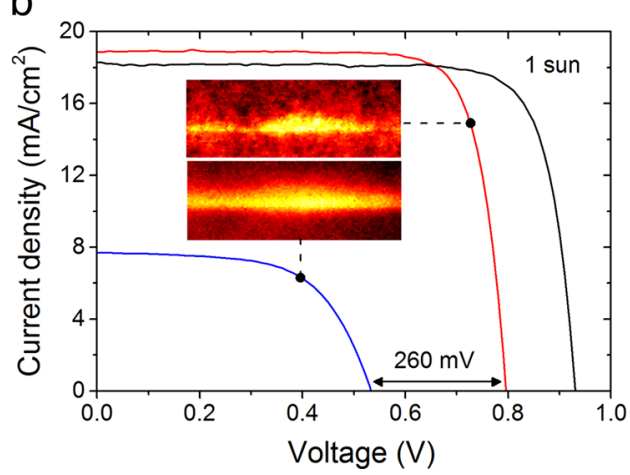

Figure 4. (a) EQE (solid lines), normalized EL spectra (dashed lines), and (b) JV curves for a GaAs control solar cell, a reference QD-IBSC and a quantum engineered QD-IBSC. Marked in (a) are the features corresponding to the InAs wetting layer $(\mathrm{WL})$ and the first quantum dot average excited state energy (e1) and the ground state transition $(\mathrm{e} 0-\mathrm{h} 0)$. The inset to $(\mathrm{b})$ shows representative quantum engineered (upper) and reference (lower) quantum dots in each device. Note that the same legend applies to (a) and (b).

and $7.7 \mathrm{~mA} / \mathrm{cm}^{-2}$ for the GaAs control, the quantum engineered QD-IBSC, and the reference QD-IBSC, respectively. These values are consistent with $J_{\mathrm{sc}}$ obtained from the integrated EQE spectra which yield 19.0, 19.2, and $11.3 \mathrm{~mA} /$ $\mathrm{cm}^{-2}$, respectively with the difference accounted for by the area of the electrical contacts. Note that these values were obtained by integrating the full spectra EQE, and while the window layer has only a small effect on $V_{\text {oc }}$ it was observed to increase $J_{\text {sc }}$ in the region $400-870 \mathrm{~nm}$ by $7.6 \mathrm{~mA} / \mathrm{cm}^{2}$.

Figure $4 \mathrm{~b}$ shows that although the quantum engineered QDIBSC exhibits lower $V_{\text {oc }}$ than the GaAs control solar cell, it has increased by a remarkable $260 \mathrm{mV}$ relative to the reference QDIBSC. It is important to note that with the exception of the window layer the devices were otherwise identical. To quantify the effect of the window layer we modeled the structures and their performance in the software SCAPS (seeFigure S7-Sn). By tuning a recombination parameter (i.e., to simulate the effect of the window layer), we matched the simulation output with the $J_{\text {sc }}$ values in Figure $4 \mathrm{~b}$. This resulted in a $V_{\text {oc }}$ difference between the reference QD-IBSC and the quantum engineered QD-IBSC of $35 \mathrm{mV}$. We can therefore be confident that the window layer has only a small effect and the increase in $V_{\mathrm{oc}}$ is mostly attributable to the nature of the quantum dot array. In summary, changing the shape and size of the quantum dots significantly affects the macroscopic performance of a QD-IBSC and in distinct contrast to other work, no strain balancing layers or high energy barriers were required to achieve this result.

The observed increase in $V_{\text {oc }}$ caused by quantum engineering the InAs quantum dot array at the nanometre scale follows directly from the creation of an IB band that is separated by a region of zero density of states from the $\mathrm{CB}$. To further examine the influence of such a band on the device performance, we measured the evolution of $V_{\text {oc }}$ for a quantum engineered QD-IBSC as a function of optical concentration ( $X$ suns) up to $X=100$ using a flash lamp solar simulator. Figure 5 a shows the difference between the open circuit voltage as a function of concentration $V_{\mathrm{oc}}(X)$ and $V_{\mathrm{oc}}(X=1)$ for a quantum engineered QD-IBSC and a high performance single energy gap GaAs concentrator solar cell. ${ }^{24}$ It is clear from Figure $5 \mathrm{a}$ that this difference increases at a faster rate (superlinearly on a logarithmic scale) for the quantum engineered QD-IBSC than for the high performance GaAs solar cell. Such superlinear behavior is the signature of three or more energy gaps in the device. On the other hand, for a conventional single gap solar cell $V_{\text {oc }} \propto(k T / q) \ln X$, that is, linearly on a log scale. In addition to the experimental data in Figure 5a, we have parametrized a drift-diffusion transport model $^{25}$ and find that the evolution of $V_{\text {oc }}$ for both the quantum engineered QD-IBSC and the GaAs concentrator are in good agreement with theory.

Having established a technique which allows for the formation of an energetically isolated IB with consequent high device $V_{\mathrm{oc}}$ we grew an additional quantum engineered QD-IBSC (Figure S3-Sn in the Supporting Information) with the objectives of further performance improvements and potential to operate under concentrated sunlight. As can be
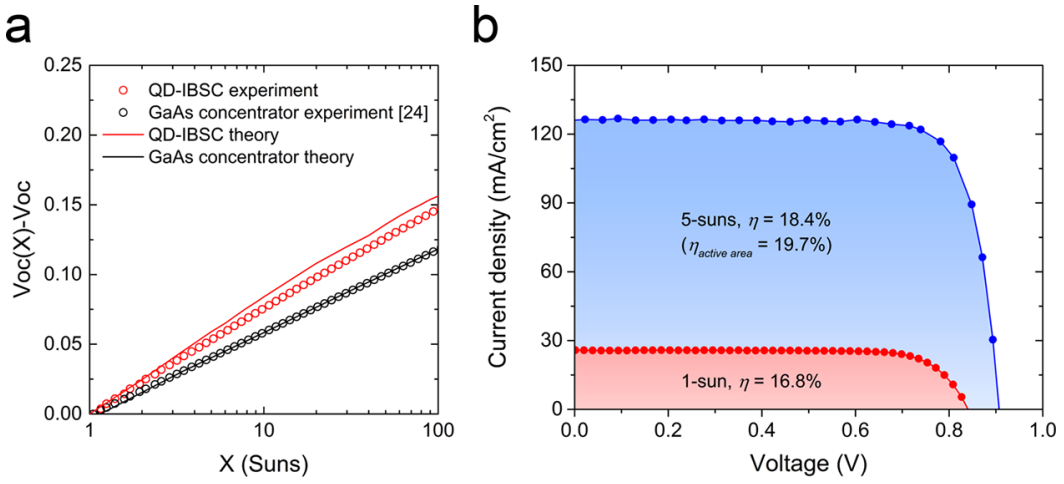

Figure 5. (a) Variation of the open circuit voltage offset, $V_{\mathrm{oc}}(X)-V_{\mathrm{oc}}(X=1)$ as a function of the light concentration $X$, for a quantum engineered QD-IBSC and a single gap conventional GaAs solar cell. Open symbols represent experiment and solid lines theory. (b) $J V$ characteristic for an optimized quantum engineered QD-IBSC at 1 and 5 suns irradiance. 
seen in Figure $5 b$, application of our quantum engineering technique increased the 1 sun $V_{\text {oc }}$ to $840 \mathrm{mV}$. Furthermore, the addition of a dual layer $\mathrm{ZnS} / \mathrm{MgF}_{2}$ antireflection coating on the surface and a back surface AlGaAs reflector resulted in $J_{\text {sc }}=26$ $\mathrm{mA} / \mathrm{cm}^{2}$. The 1 sun $J V$ characteristic shown in Figure $5 \mathrm{~b}$ is taken from one of five devices from the same device processing batch. The high uniformity of our devices is demonstrated by average values of $V_{\mathrm{oc}}=822 \pm 14 \mathrm{mV}, J_{\mathrm{sc}}=25.9 \pm 0.2 \mathrm{~mA} / \mathrm{cm}^{2}$, fill factor $\mathrm{FF}=76.3 \pm 1.2$, and efficiency $\eta=16.3 \pm 0.5 \%$. The equivalent parameter set for seven GaAs control solar cells from the same processing batch is $V_{\mathrm{oc}}=891 \pm 60 \mathrm{mV}, J_{\mathrm{sc}}=25.0 \pm$ $1.27 \mathrm{~mA} / \mathrm{cm}^{2}$, fill factor $\mathrm{FF}=75.3 \pm 2.0$, and efficiency $\eta=16.7$ $\pm 1.2 \%$.

A key challenge for InAs QD-IBSCs has been to achieve $V_{\text {oc }}$ values that are comparable with GaAs control devices that do not contain quantum dots. Interestingly, within the limit of our sample size and the experimental uncertainty, our results show that we can use quantum engineering to obtain a $V_{\text {oc }}$ that matches the performance of a GaAs control. Another approach that has been used successfully to increase $V_{\mathrm{oc}}$ is the addition of wider band gap barrier layers such as $\mathrm{AlGaAs}^{26,27}$ and GaAsP. ${ }^{10}$ Notably, the latter resulted in $V_{\text {oc }} \sim 1 \mathrm{~V}$, however, the inclusion of multiple layers of wider band gap semiconductors will significantly alter the energy band structure and the validity of comparisons with QD-IBSCs (or indeed GaAs control solar cells) that do not contain these layers is unclear. Two notable values of $V_{\text {oc }}$ achieved for InAs QD-IBSC without additional barrier layers are $840^{28}$ and $873 \mathrm{mV}^{29}$ While these are comparable to our values, they were obtained using fewer stacked layers in the quantum dot array. This is an important distinction because although $V_{\mathrm{oc}}$ has been observed to increase with fewer quantum dot layers, ${ }^{30}$ a high number of layers is desirable in order to maximize the additional photocurrent generated by the IB. The distinctive benefit therefore of our quantum engineering approach is that it increases both $V_{\mathrm{oc}}$ and $J_{\text {sc }}$ simultaneously.

It is important to note that a high $V_{\mathrm{oc}}$ follows naturally from the formation and operation of a true IB and our work is the first to focus on this aspect of the InAs QD-IBSC. By characterizing the geometry and dimensions of individual quantum dots in the array at the nanoscale, we have demonstrated the opening of a clear second energy gap of the order $90 \mathrm{meV}$. Our calculations ${ }^{21}$ indicate that this could be increased to $\sim 120 \mathrm{meV}$ if the average quantum dot diameter could be further reduced to $110 \AA$, which should be within practical MBE growth limits.

The real power of the IBSC becomes clear under concentrated sunlight where not only does $V_{\text {oc }}$ increase at a superior rate to a single energy gap device (see Figure 5a), but so also does $J_{\mathrm{sc}}$. This is because the additional photocurrent resulting from the $\mathrm{IB}^{31}$ has a quadratic dependence on the incident power. ${ }^{32}$ Taken together these factors offer the potential for ultrahigh efficiency under concentration. We have previously reported $V_{\mathrm{oc}}=985 \mathrm{mV}$ at 500 suns obtained using suns $-V_{\text {oc }}$ measurements. Similarly, other groups ${ }^{9,30}$ have also reported strong potential for the technology under concentration, however, there is a striking lack of $J V$ data. Figure $5 \mathrm{~b}$ shows the $J V$ curve for a quantum engineered $\mathrm{QD}$ IBSC at 5 suns obtained using a flash-lamp simulator which was cross-calibrated with the 1 sun solar simulator for accuracy. The results show excellent performance up to 5 suns concentration with little degradation of the fill factor and analysis of the evolution of $V_{\text {oc }}$ with concentration indicates the diode ideality factor to be $n=1.2$. The diode ideality factor was determined by fitting the function $V_{\mathrm{oc}}(X)=V_{\mathrm{ocl}}+\left(n k_{\mathrm{B}} T / q\right) \ln X$ to the evolution of $V_{\mathrm{oc}}$ with concentration ( $X$ suns), as shown in Figure S5-Sn. In this expression, $V_{\mathrm{oc}}(X)$ is the open circuit voltage at $X$ suns concentration and $V_{\mathrm{ocl}}$ is the one sun open circuit voltage. This is further evidence of the material quality as it approaches the ideal value of $n=1$ for any optoelectronic $p n$ junction device. The efficiency of the device shown in Figure $5 \mathrm{~b}$ is $\eta=18.4 \%$, which increases to a record efficiency under concentration of $19.7 \%$ when the area of the contacts is taken into account (the active area efficiency). To the best of our knowledge, this is the highest reported efficiency for a InAs QD-IBSC. Beyond 5 suns, the fill factor starts to degrade thereby offsetting the performance improvement from the IB and current research efforts should be focused on incorporating an IB into a optimized concentrating solar cell with appropriate electrical contact geometry.

We have presented a novel approach, based on quantum engineering, to the formation of an IB of states within a GaAs solar cell using an InAs quantum dot array. By precisely engineering the shape and size of the quantum dots within the array it is possible to create an IB of states which is energetically isolated from the CB. Calculations based on experimentally obtained quantum dot dimensions show that this opens up a clear second energy gap in the device which leads directly to high $V_{\text {oc }}$. The results demonstrate that nanometre variations in the material used to create the IB have a profound effect on the macroscopic device performance. Therefore, in order to progress the technology, we propose that it is necessary to quantum mechanically design and engineer the InAs quantum dot array. Under concentrated sunlight the benefits of the IB become disproportionately large and experimental results from quantum engineered QD-IBSCs exhibit new levels of photovoltaic performance.

\section{ASSOCIATED CONTENT}

\section{Supporting Information}

The Supporting Information is available free of charge on the ACS Publications website at DOI: 10.1021/acsphotonics.7b00673.

Device structures, characterization techniques, concentration measurements and analysis, and device simulations (PDF).

\section{AUTHOR INFORMATION}

\section{Corresponding Authors}

*E-mail: neil.beattie@northumbria.ac.uk.

*E-mail: s.tomic@salford.ac.uk.

ORCID ब

Stanko Tomić: 0000-0003-3622-6960

Notes

The authors declare no competing financial interest.

\section{ACKNOWLEDGMENTS}

The authors are grateful to Alex Savidis and Solar Capture Technologies Ltd. for providing access to the flash lamp solar simulator used in the concentration measurements. S.T. wishes to thank the Royal Society, London for the Grant "High Performance Computing in Modelling of Innovative Photovoltaic Devices" and also the contribution of EU COST Action MP1406. The authors also acknowledge financial support from 
the European Union under the Seventh Framework Programme under a contract for an Integrated Infrastructure Initiative, Reference 312483-ESTEEM2.

\section{REFERENCES}

(1) Green, M. A. Third generation photovoltaics: Ultra-high conversion efficiency at low cost. Prog. Photovoltaics 2001, 9, 123-135.

(2) Shockley, W.; Queisser, H. J. Detailed balance limit of efficiency of $p$-n junction solar cells. J. Appl. Phys. 1961, 32, 510.

(3) Luque, A.; Martí, A. Increasing the efficiency of ideal solar cells by photon induced transitions at intermediate levels. Phys. Rev. Lett. 1997, 78, 5014-5017.

(4) Okada, Y.; Ekins-Daukes, N. J.; Kita, T.; Tamaki, R.; Yoshida, M.; Pusch, A.; Hess, O.; Phillips, C. C.; Farrell, D. J.; Yoshida, K.; Ahsan, N.; Shoji, Y.; Sogabe, T.; Guillemoles, J. F. Intermediate band solar cells: Recent progress and future directions. Appl. Phys. Rev. 2015, 2, 021302 .

(5) Tomić, S. Intermediate-band solar cells: Influence of band formation on dynamical processes in InAs/GaAs quantum dot arrays. Phys. Rev. B: Condens. Matter Mater. Phys. 2010, 82, 195321.

(6) Zhou, D.; Vullum, P. E.; Sharma, G.; Thomassen, S. F.; Holmestad, R; Reenaas, T. W.; Fimland, B. O. Positioning effects on quantum dot solar cells grown by molecular beam epitaxy. Appl. Phys. Lett. 2010, 96, 83108.

(7) Wu, J.; Makableh, F. M.; Vasan, R.; Manasreh, M. O.; Liang, B.; Reyner, C. J.; Huffaker, D. L. Strong interband transitions in InAs quantum dots solar cell. Appl. Phys. Lett. 2012, 100, 51907.

(8) Antolín, E.; Martí, A.; Farmer, C. D.; Linares, P. G.; Hernández, E.; Sánchez, A. M.; Ben, T.; Molina, S. I.; Stanley, C. R.; Luque, A. Reducing carrier escape in the InAs/GaAs quantum dot intermediate band gap solar cell. J. Appl. Phys. 2010, 108, 64513.

(9) Tanabe, K.; Guimard, D.; Bordel, D.; Arakawa, Y. High-efficiency InAs/GaAs quantum dot solar cells by metal organic chemical vapor deposition. Appl. Phys. Lett. 2012, 100, 193905.

(10) Bailey, C. G.; Forbes, D. V.; Raffaelle, R. P.; Hubbard, S. M. Near $1 \mathrm{~V}$ open circuit voltage InAs/GaAs quantum dot solar cells. Appl. Phys. Lett. 2011, 98, 163105.

(11) Willis, S. M.; Dimmock, J. A. R.; Tutu, F.; Liu, H. Y.; Peinado, M. G.; Assender, H. E.; Watt, A. A. R.; Sellers, I. R. Defect mediated extraction in InAs/GaAs quantum dot solar cells. Sol. Energy Mater. Sol. Cells 2012, 102, 142-147.

(12) Jolley, G.; Lu, H. F.; Fu, L.; Tan, H. H.; Jagadish, C. Electronhole recombination properties of $\operatorname{In}_{0.5} \mathrm{Ga}_{0.5} \mathrm{As} / \mathrm{GaAs}$ quantum dot solar cells and the influence on the open circuit voltage. Appl. Phys. Lett. 2010, 97, 123505 .

(13) Tomić, S.; Sogabe, T.; Okada, Y. In-plane coupling effect on absorption coefficients of InAs/GaAs quantum dot arrays for intermediate band solar cell. Prog. Photovoltaics 2015, 23, 546-558.

(14) Sogabe, T.; Shoji, Y.; Ohba, M.; Yoshida, K.; Tamaki, R.; Hong, H.-F.; Wu, C.-H.; Kuo, C.-T.; Tomić, S.; Okada, Y. Intermediate-band dymanics of quantum dots solar cell in concentrator photovoltaic modules. Sci. Rep. 2015, 4, 4792.

(15) Kerestes, C.; Polly, S.; Forbes, D.; Bailey, C.; Podell, A.; Spann, J.; Patel, P.; Richards, B.; Sharps, P.; Hubbard, S. Fabrication and analysis of multijunction solar cells with a quantum dot (In)GaAs junction. Prog. Photovoltaics 2014, 22, 1172-1179.

(16) Nuntawong, N.; Tatebayashi, J.; Wong, P. S.; Huffaker, D. L. Localized strain reduction in strain-compensated InAs/GaAs stacked quantum dot structures. Appl. Phys. Lett. 2007, 90, 163121.

(17) Oshima, R.; Takata, A.; Okada, Y. Strain-compensated InAs/ GaNAs quantum dots for use in high-efficiency solar cells. Appl. Phys. Lett. 2008, 93, 83111.

(18) Haffouz, S.; Raymond, S.; Lu, Z. G.; Barrios, P. J.; Roy-Guay, D.; Wu, X.; Liu, J. R.; Poitras, D.; Wasilewski, Z. R. Growth and fabrication of quantum dots superluminescent diodes using the indium-flush technique: A new approach to controlling the bandwidth. J. Cryst. Growth 2009, 311, 1803-1806.
(19) Keizer, J. G.; Clark, E. C.; Bichler, M.; Abstreiter, G.; Finley, J. J.; Koenraad, P. M. An atomically resolved study of InGaAs quantum dot layers grown with an indium flush step. Nanotechnology 2010, 21, 215705 .

(20) Rosenauer, A.; Mehrtens, T.; Müller, K.; Gries, K.; Schowalter, M.; Satyam, P. V.; Bley, S.; Tessarek, C.; Hommel, D.; Sebald, K.; Seyfried, M.; Gutowski, J.; Avramescu, A.; Engl, K.; Lutgen, S. Composition mapping in InGaN by scanning transmission electron microscopy. Ultramicroscopy 2011, 111, 1316-1327.

(21) Tomić, S.; Jones, T. S.; Harrison, N. M. Absorption characteristics of a quantum dot array induced intermediate band: Implications for solar cell design. Appl. Phys. Lett. 2008, 93, 263105.

(22) Luque, A.; Martí, A.; Stanley, C. Understanding intermediate band solar cells. Nat. Photonics 2012, 6, 146-152.

(23) Beattie, N. S.; Zoppi, G.; See, P.; Farrer, I.; Duchamp, M.; Morrison, D. J.; Miles, R. W.; Ritchie, D. A. Analysis of InAs/GaAs quantum dot solar cells using suns-Voc measurements. Sol. Energy Mater. Sol. Cells 2014, 130, 241-245.

(24) MacMillan, H. F.; Hamaker, H. C.; Kaminar, N. R.; Gee, J. M.; Kuryla, M. S.; Ladle, M.; Liu, D. D.; Virshup, G. F. $28 \%$ efficient GaAs concentrator solar cells. Proceedings of the 20th IEEE Photovoltaics Specialists Conference; IEEE, 1988.

(25) Strandberg, R.; Reenaas, T. W. Photofilling of intermediate bands. J. Appl. Phys. 2009, 105, 124512.

(26) Varghese, A.; Yakimov, M.; Tokranov, V.; Mitin, V.; Sablon, K.; Sergeev, A.; Oktyabrsky, S. Complete voltage recovery in quantum dot solar cells due to suppression of electron capture. Nanoscale 2016, 8, 7248.

(27) López, E.; Datas, A.; Ramiro, I.; Linares, P. G.; Antolín, E.; Artacho, I.; Martí, A.; Luque, A.; Shoji, Y.; Sogabe, T.; Ogura, A.; Okada, Y. Demonstration of the operation principles of intermediate band solar cells at room temperature. Sol. Energy Mater. Sol. Cells 2016, $149,15-18$.

(28) Blokhin, S. A.; Sakharov, A. V.; Nadtochy, A. M.; Pauysov, A. S.; Maximov, M. V.; Ledentsov, N. N.; Kovsh, A. R.; Mikhrin, S. S.; Lantratov, V. M.; Mintairov, S. A.; Kaluzhniy, N. A.; Shvarts, M. Z. AlGaAs/GaAs photovoltaic cells with an array of InGaAs QDs. Semiconductors 2009, 43, 514-518.

(29) Guimard, D.; Morihara, R.; Bordel, D.; Tanabe, K.; Wakayama, Y.; Nishioka, M.; Arakawa, Y. Fabrication of InAs/GaAs quantum dot solar cells with enhanced photocurrent and without degradation of open circuit voltage. Appl. Phys. Lett. 2010, 96, 203507.

(30) Hubbard, S. M.; Bailey, C. G.; Aguinaldo, R.; Polly, S.; Forbes, D. V.; Raffaelle, R. P. Characterization of quantum dot enhanced solar cells for concentrator photovoltaics. Proceedings of the 34th IEEE Photovoltaics Specialists Conference; IEEE, 2009.

(31) Datas, A.; López, E.; Ramiro, I.; Antolí, E.; Martí, A.; Luque, A.; Tamaki, R.; Shoji, Y.; Sogabe, T.; Okada, Y. Intermediate band solar cell with extreme broadband spectrum quantum efficiency. Phys. Rev. Lett. 2015, 114, 157701.

(32) Li, T.; Dagenais, M. Non-reasonant below-bandgap two-photon absoprtion in quantum dot solar cells. Appl. Phys. Lett. 2015, 106, 171101. 